Wilfrid Laurier University

Scholars Commons @ Laurier

Physics and Computer Science Faculty

Publications

Physics and Computer Science

10-1989

\title{
Solution Deposited Optical Waveguide Lens
}

Daniel W. Hewak

University of Waterloo

John W.Y. Lit

Wilfrid Laurier University, jlit@wlu.ca

Follow this and additional works at: https://scholars.wlu.ca/phys_faculty

\section{Recommended Citation}

Hewak, Daniel W. and Lit, John W.Y., "Solution Deposited Optical Waveguide Lens" (1989). Physics and Computer Science Faculty Publications. 13.

https://scholars.wlu.ca/phys_faculty/13

This Article is brought to you for free and open access by the Physics and Computer Science at Scholars Commons @ Laurier. It has been accepted for inclusion in Physics and Computer Science Faculty Publications by an authorized administrator of Scholars Commons @ Laurier. For more information, please contact scholarscommons@wlu.ca. 


\title{
Solution deposited optical waveguide lens
}

\author{
Daniel W. Hewak and John W. Y. Lit
}

\begin{abstract}
The development of a solution deposited optical waveguide lens, whose focusing effect is produced by an effective index gradient with a hyperbolic secant variation, is described. The lenses are fabricated by a microcontrolled dip coating procedure from colloidal $\mathrm{SiO}_{2}: \mathrm{TiO}_{2}$ solutions. Both the design and construction of the lens, along with the analytical and experimental results of the focusing properties, are described. The best lenses had speeds of $\sim f / 10$ and focal spots $\sim 1.2$ times the diffraction limit at apertures of $2.0 \mathrm{~mm}$.
\end{abstract}

\section{Introduction}

Almost exclusively in the past, thin film lenses which focus by virtue of an effective index gradient have been fabricated by sputtering or evaporation through an appropriately shaped mask. By far, the Luneburg lens has received the most attention since its first demonstration in thin film form by Zernike. ${ }^{1}$ Since then, Yao et al. ${ }^{2}$ have developed a computational procedure to derive the film thickness and profile required to achieve a desired focusing effect. They reproducibly obtained nearly diffraction limited performance for a family of circular Luneburg lenses ranging between $f / 4$ and $f / 30$ when only the center portion of the lens aperture was utilized. Wood et al ${ }^{3}$ fabricated rectangular Luneburg-type lenses by thermal evaporation of $\mathrm{As}_{2} \mathrm{~S}_{3}$ through suitable masks onto single-mode $\mathrm{LiNbO}_{3}$ waveguides. The best lenses had speeds of $f / 5.5$ at an aperture of $10 \mathrm{~mm}$ and focal spots at an aperture reduced to $3 \mathrm{~mm}$ of 1.2 times the diffraction limit. Anderson et al. ${ }^{4}$ included a Luneburg beam expansion lens system in an integrated optical spectrum analyzer, which was fabricated by sputtering through a mask onto a $\mathrm{SiO}_{2}$ isolation layer grown on a Si substrate. Similarly, Bryan ${ }^{5}$ developed a $\mathrm{Ta}_{2} \mathrm{O}_{5}$ lens for use in a wavelength division multiplexer.

Deposition from the solution by microprocessor controlled dip coating is a novel fabrication procedure which we use to fabricate a different type of planar thin

Daniel Hewak is with University of Waterloo, Guelph-Waterloo Program for Graduate Work in Physics, Waterloo, Ontario N2L 3G1, Canada, and John Lit is with Wilfrid Laurier University, Department of Physics \& Computing, Waterloo, Ontario N2L 3C5, Canada.

Received 28 October 1988.

0003-6935/89/194190-09\$02.00/0.

(C) 1989 Optical Society of America. film lens. The concept of thickness varying structures deposited by solution was introduced in an earlier work. ${ }^{6}$ By carefully controlling the withdrawal speed of a substrate from solution, we achieved thin films with smooth and continuous thickness changes. By profiling the thickness of a thin film waveguide in a precise manner, we achieved an effective index gradient which is suitable for focusing and collimating guided waves.

The thickness gradients are linear and parallel to the direction of withdrawal of the substrate from the solution. Thus our lenses are restricted to those with axial symmetry. However, it is well known that an index gradient with a hyperbolic secant distribution, which can be obtained with such a thickness gradient, has the potential to provide perfect focusing. Thus it is our goal to fabricate a solution deposited hyperbolic secant lens.

We have used commercial colloidal $\mathrm{SiO}_{2}$ and $\mathrm{TiO}_{2}$ solutions which yield thin films with a refractive index of 1.4-2.1, depending on the relative concentrations of the two materials. A single dip coating typically yields a thin film that is $0.05-2.5 \mu$ m thick. ${ }^{7}$

We begin this paper in Sec. II by briefly outlining the theory of the hyperbolic secant lens. This introduces the concept of this focusing structure and the focusing properties that can be expected. In Sec. III we describe the experimental fabrication and development of the lens. The use of a microcontrolled dip coating to provide thickness varying structures is briefly outlined, and the method used to achieve a lens based on the hyperbolic secant gradient is described. Also here we undertake a ray tracing analysis of the lens structure, determining the expected focusing properties of the profiles we have fabricated. Finally, in Sec. IV the lens prototypes we have fabricated are experimentally studied. A guided wave is coupled to the lens structure, and the focal length and intensity pattern in the focal plane are measured. 
a.

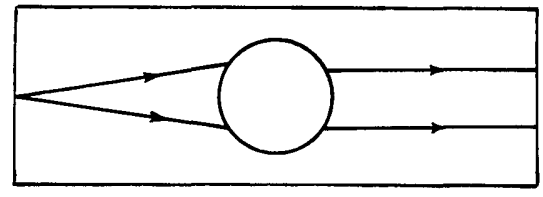

b.

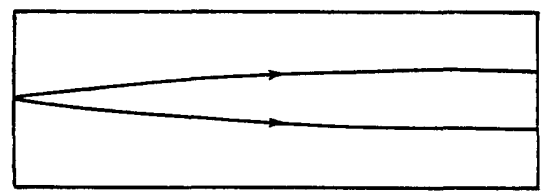

Fig. 1. Top view of the planar optical waveguide lens illustrating (a) a conventional structure of a localized lens and (b) a proposed lens based on an axial index gradient.

\section{Theory of the Hyperbolic Secant Lens}

Conventional optical waveguide lenses, namely, the mode index, geodesic, and grating lens, ${ }^{8}$ are localized structures surrounded by a planar waveguide which interconnects the lens with a waveguide edge or other integrated components. Although most of these lenses can provide diffraction limited performance, which in one sense makes them ideal focusing structures, there is still room for improvement. In many applications of the optical waveguide lens, such as an integrated optical rf spectrum analyzer or a wavelength division multiplexer, the image and/or object planes coincide with a waveguide edge. In these cases, the lens either focuses a guided wave to a point for coupling into a fiber or channel waveguide or collimates a point source located on the waveguide edge for propagation through the waveguide. In these cases, a localized lens structure, as shown in Fig. 1(a), can in fact contribute to insertion losses due to mode conversion and scattering at the lens edge. ${ }^{9}$

By using a lens based on an axial index gradient, a rectangular lens, as shown in Fig. 1(b), may be achieved. With the proper choice of focal length, such focusing can extend from waveguide edge to waveguide edge, eliminating two dielectric boundaries that would otherwise be found in a localized lens and thus potentially improving lens performance.

It is well known that the distribution ${ }^{10-12}$

$$
n(x)=n_{0} \operatorname{sech}(g x)
$$

provides perfect focusing. Over a distance $L=\pi / g$, this gradient will refocus a point source exactly to a point. Over one-half of this distance, $L=\pi / 2 g$, this gradient will serve to collimate a point source or focus a parallel beam exactly to a point, as shown in Fig. 1(b). We have verified that materials exist for the realization of practical hyperbolic secant lenses, with focal lengths and $f /$ Nos. that would be useful for guided wave applications. The effective index gradient could be achieved by appropriately profiling the thickness of either the waveguide or an additional overlay layer, as is done for the thin film Luneburg lens. ${ }^{1-5}$ Figure 2 illustrates the thickness profiles of a family of hyper-

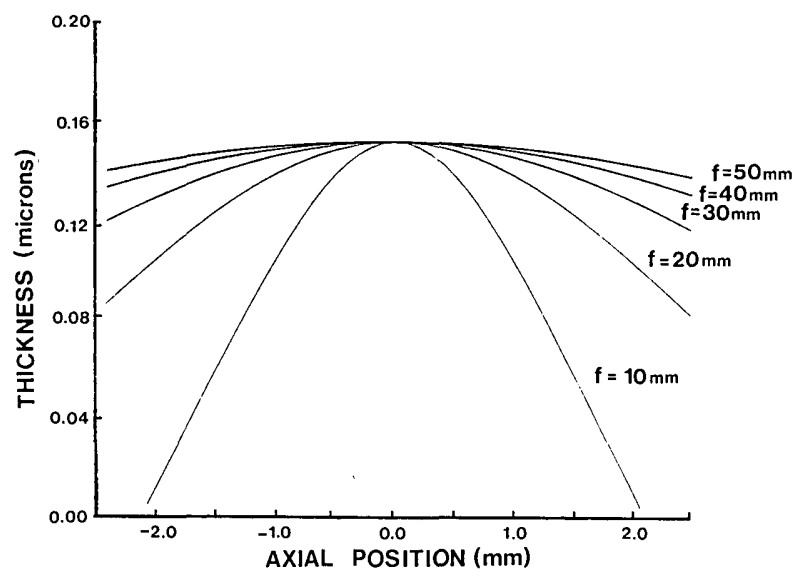

Fig. 2. Thickness profiles for a family of hyperbolic secant lenses with focal lengths of 10-50 mm and apertures of $5 \mathrm{~mm}$ (design parameters: $n_{1}=1.522, n_{2}=1.80, n_{3}=1.80, n_{4}=1.0, d_{1}=0.2 \mu \mathrm{m}$, $\lambda=0.633 \mu \mathrm{m})$.

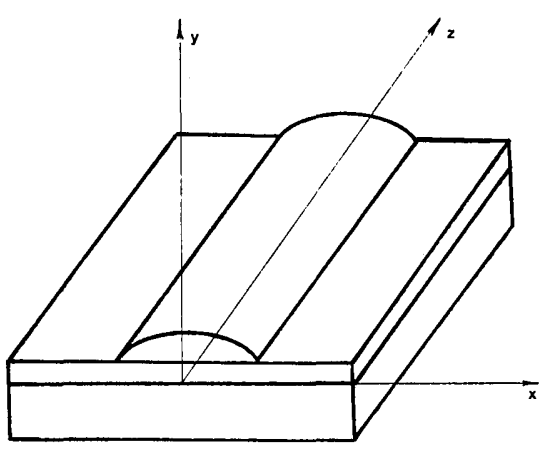

Fig. 3. Geometry and coordinate system used in the analysis of the hyperbolic secant lens.

bolic secant lenses. For such the thickness change over a distance of one wavelength is negligible. Thus the geometrical optics and ray tracing is a valid and useful tool for evaluation of the hyperbolic secant lens.

Figure 3 shows the geometry and coordinate system we use in an analysis of such a lens. The starting point in any ray analysis of an inhomogeneous medium is the ray equation

$$
\partial / \partial s(n \partial \bar{r} / \partial s)=\nabla n
$$

from which the ray position vector $\bar{r}$ and the ray direction vector $\bar{s}$ are determined for points along the trajectory of a ray. Here $d s$ is an increment of the physical path along the ray, and $n$ is the local effective or refractive index.

A complete solution of the ray equation for many index distributions can be found in the textbook by Marchand. ${ }^{13}$ For an axial index gradient, with propagation in the $z$ direction, the ray equation reduces to

$$
\frac{d^{2} \vec{r}}{d z^{2}}=(1 / n) \frac{d n}{d x} .
$$

For the 2-D planar waveguide, Marchand has shown that when $n(x)$ is given by Eq. (1), the solutions of Eq. (3) have the form 


$$
\sinh (g x)=A \sin \left[B \pm g\left(z-z_{0}\right)\right]
$$

where

$$
\begin{aligned}
& A=\left[\left(n_{0} / q_{0}\right)^{2}-1\right]^{1 / 2}, \\
& B=\sin ^{-1}\left[(1 / A) \sinh \left(g x_{0}\right)\right],
\end{aligned}
$$

and the initial direction cosines are

$$
\begin{aligned}
& p_{0}=n\left(x_{0}\right) \sin \theta_{0}, \\
& q_{0}=n\left(x_{0}\right) \cos \theta_{0} .
\end{aligned}
$$

For an axial index distribution, $q=q_{0}$ is a constant along a ray path. Given the initial ray position $\left(x_{0}, z_{0}\right)$ and initial direction $\theta_{0}$, Eq. (4) can be used to determine any point on the ray path.

It is apparent from this equation that every ray repeats itself periodically with a period, $L=2 \pi / g$, which is independent of the ray or the starting position. This confirms the exact focusing to a point which is expected.

The numerical aperture (N.A.) or light gathering ability of a lens is an important consideration in many applications. When coupling to and from the lens, mismatch between the N.A. of the lens and the coupling component can be a significant source of loss. ${ }^{14}$ For example, loss results when the light emitted from a light source is restricted by the N.A. of the lens or when the focused light is coupled to an optical fiber with a N.A. less than that of the lens.

The numerical aperture of a hyperbolic secant lens in the plane of the lens can be determined by considering the maximum angle $\theta_{a}$ at which a ray can be accepted by the lens aperture and still remain focused:

$$
\text { N.A. }=\sin \theta_{a}=\left(1 / n_{a}\right) n\left(x_{0}\right) \sin \theta .
$$

Here the lens is surrounded by a medium of refractive index $n_{a}$, and $x_{0}$ and $\theta_{0}$ are the initial position and direction of the ray in the aperture of the lens. Setting $x=a$ in Eq. (4), where $a$ is the $x$ coordinate of the lens aperture, we can calculate the maximum initial angle $\theta_{0}$ as a function of $x_{0}$, which would allow a ray to propagate through the lens without vignetting. This yields

$$
\text { N.A. }=\left(1 / n_{a}\right)\left[n^{2}\left(x_{0}\right)-n^{2}(a)\right]^{1 / 2} .
$$

It is apparent from this equation that the N.A. is a maximum at the lens axis and decreases to zero at the edge of the aperture. This is expected for a gradient index lens.

For a typical lens, $a=5.0 \mathrm{~mm}, g=\pi / 50 \mathrm{~mm}^{-1}, n_{0}=$ 1.5 , and we can assume that the lens is surrounded by air. Equation (10) then yields a maximum N.A. of 0.47 , which corresponds to an acceptance angle of $28^{\circ}$. Such a value is comparable to the N.A. of a GRIN rod lens of similar dimensions.

\section{Lens Fabrication and Development}

In this section we begin experimental development of the hyperbolic secant lens. Fabrication is obtained by deposition from a solution using a novel microprocessor controlled dip coating procedure. By profil- ing the thickness of a thin film optical waveguide, we hope to achieve an effective index gradient with a hyperbolic secant distribution, which is suitable for focusing and collimating guided waves.

\section{A. Microcontrolled Dip Coating}

The details of a typical dip coating procedure for the fabrication of planar optical waveguides have been described elsewhere. ${ }^{14,15}$ Briefly, substrates are lowered into the solution and withdrawn vertically with a smooth and constant velocity. The films are dried while in a vertical position for a few minutes at $\sim 100^{\circ} \mathrm{C}$ and then baked at a high temperature, usually up to the strain point of the substrate, to harden the film and drive off any solvents remaining in the waveguiding material.

We have utilized a $\mathrm{SiO}_{2}: \mathrm{TiO}_{2}$ commercial solution for our application in the development of our solution deposited lens. The resulting waveguides have proved to be of good optical quality, being hard, resistant, and with low losses. These optical properties have been previously discussed in detail. Briefly, Tiefenthaler et al. ${ }^{14}$ measured losses with a light scattering method and by examining the attenuation between prism incoupling and outcoupling. Losses varied from 0.21 to $6.61 \mathrm{~dB} / \mathrm{cm}$, depending on the wavelength, $\mathrm{TiO}_{2}$ content and the method of measurement.

The principle of obtaining thickness varying structures by varying the dipping speed was first demonstrated in an earlier work. ${ }^{6}$ A microprocessor, which forms the basis of the instrument, can be programmed through a keyboard and screen with a series of commands which define a lifting speed and time interval for which this lifting speed is to be maintained. Execution of these commands in succesive order results in a predetermined lifting speed pattern being sent to the dipping motor via a digital-to-analog converter. The D-A converter transforms the digital signals of the microprocessor to discrete voltages. The lifting speed of the motor is proportional to the applied voltage. Thus the result is a series of discrete lifting speeds. We have used an 8-bit D-A converter which provides 256 discrete lifting speeds ranging from $\sim 50$ to 250 $\mathrm{mm} / \mathrm{min}$. Although the digital signals sent to the dipping motor are discrete and changed abruptly, the momentum of the motor and the flowing nature of the deposition process smooth out the voltage and withdrawal speed changes, resulting in a smooth and continuous change in thickness.

Before describing the procedure for lens fabrication, a few general comments can be made. First and most important, the measured thickness changes are smooth and continuous. We observed very few major irregularities such as flow lines or abrupt thickness changes in the thickness profiles measured. Any thickness irregularities observable in the profiles were of the order of $0.001 \mu \mathrm{m}$, which is comparable to the accuracy obtained with the ellipsometer. Second, film shrinkage during drying and baking was relatively independent of initial wet film thickness and thus had only a small effect of the final film profile. A taper 
that was deposited with a linear change in speed during deposition had a linear thickness change following heat treatment. This important property, that of a shrinkage relatively independent of initial film thickness, was also observed by Heuberger and Lukosz ${ }^{16}$ in their development of an embossing technique for these solutions.

\section{B. Fabrication}

The procedure for depositing thin film lenses by microprocessor controlled dip coating is similar to that for other thickness varying structures with one important difference. We are now much more concerned with duplicating a precise profile with thickness changes of the order of a few nanometers per millimeter along the substrate. Now, to achieve a desired hyperbolic secant effective index gradient, both absolute and relative thickness changes must be carefully reproduced so that aberrationless focusing is obtained. Figure 2 illustrates the thickness profiles required for a typical family of lenses with focal lengths of $10-50 \mathrm{~mm}$ and widths of $5 \mathrm{~mm}$. These lenses are designed for $\lambda=$ $0.633 \mu \mathrm{m}$ for fabrication on Corning 7059 substrates $(n$ $=1.522$ ) from a solution yielding a refractive index of 1.8 on a planar waveguide $0.2 \mu \mathrm{m}$ thick with air as a cover.

We will concentrate our efforts here on the fabrication of lenses with focal lengths of the order of $f \sim 20$ $\mathrm{mm}$, although other lens dimensions could be fabricated with the same procedure. Lenses with widths up to $\sim 15 \mathrm{~mm}$ will be deposited. However, we expect usable apertures to be only a few millimeters. These dimensions are chosen only for convenience, as they correspond to the substrate sizes that we have.

The first step in depositing a lens is to determine the withdrawal speed pattern required to achieve the desired thickness profile. With our prior knowledge of the dependence of thickness on the withdrawal rate for planar waveguides, as shown in Fig. 4, we can determine an approximate withdrawal speed pattern required to achieve a desired lens. If the linear response between withdrawal rate and thickness observed for planar guides also applied to thickness varying structures, the withdrawal speeds required to achieve this lens would be proportional to the desired thickness profile. However, as we found when we were depositing tapers, this linear response is not obtained for thickness varying structures. The combined effects of gravity and solution viscosity result in a nonlinear relationship between withdrawal rate and thickness for thickness varying structures.

None the less, Fig. 4 is a good starting point for determining the required lifting speed pattern. Using these curves as a guide to estimating the withdrawal speeds required for the planar region surrounding the lens and the lens peak thickness, we have written a control program for the microprocessor controlled dipping arm, which sends the withdrawal speed pattern illustrated in Fig. 5 to the dipping arm motor.

Having determined the withdrawal speed pattern which will be used to fabricate the thickness varying

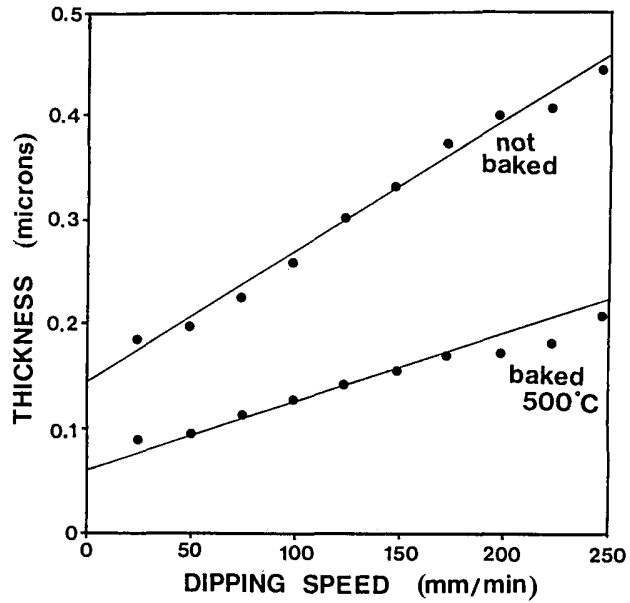

Fig. 4. Thin film thickness as a function of the withdrawal speed for a $\mathrm{SiO}_{2}: \mathrm{TiO}_{2}$ solution which yields $n=1.8$.

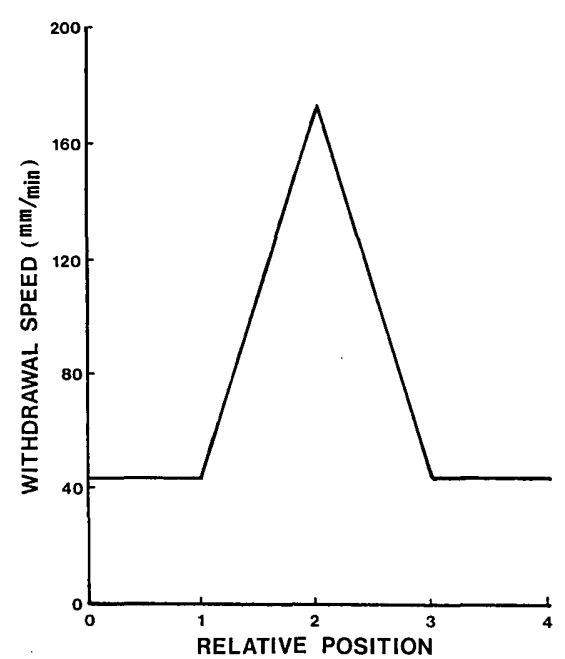

Fig. 5. Withdrawal speed pattern used in an attempt to achieve a lens with focal length $f=20 \mathrm{~mm}$.

layer of the lens, the procedure is now as follows. A planar waveguide is first deposited with a thickness of $0.2 \mu \mathrm{m}$ from Liquicoat solution yielding $n=1.8$. This could be deposited from either a single dip with a withdrawal rate of $225 \mathrm{~mm} / \mathrm{min}$ or two dips at $64 \mathrm{~mm} /$ min. Heat treatment is typically drying for $15 \mathrm{~min}$ at $100^{\circ} \mathrm{C}$ followed by baking for $1 \mathrm{~h}$ at $500^{\circ} \mathrm{C}$ and is performed after each dipping. Following deposition of the planar guide, the thickness varying region is deposited, using microprocessor control to provide the withdrawal speed pattern given by Fig. 5 . Heat treatment is the same as that for the planar guide.

The thickness profile of the resulting structure was measured using ellipsometry at $200-\mu \mathrm{m}$ intervals across the thickness varying region and is illustrated in Fig. 6. The effects of gravity on profile symmetry are immediately evident. Also we note that the peak thickness of $0.295 \mu \mathrm{m}$ is almost double the expected peak thickness. There is also some irregularity in the thickness profile near the region of maximum thick- 


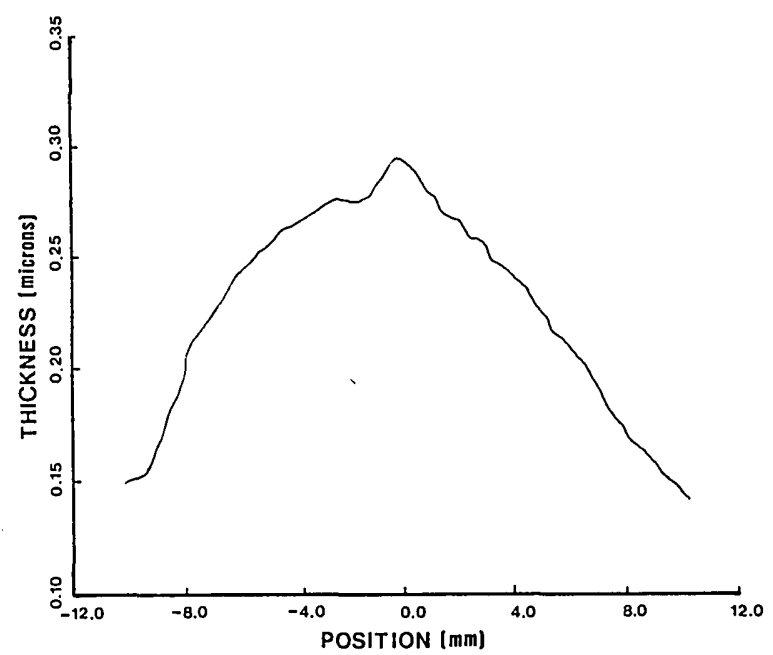

Fig. 6. Thickness profile of the first lens attempt fabricated with the withdrawal pattern illustrated in Fig. 5 .

ness. From this result it was apparent that, at least initially, determining the optimum withdrawal pattern for a desired lens would initially be largely a matter of trial and error. The linear relationship between withdrawal speed and resulting film thickness, which was found for planar thin films, did not apply to film structures with varying thickness.

Over the course of $\sim 1$ yr, a number of lens profiles were deposited and evaluated. In the early stages of lens fabrication, it was sufficient to observe the colored interference bands in the thickness varying region of the waveguide, which were most visible when the slide was observed with reflected white light prior to baking. An asymmetric or poor quality lens could then be immediately detected by the difference in width of these colored bands and the withdrawal speed pattern appropriately modified.

The major hurdle in developing a practical lens was compensating for the effects of gravity. As an aid to overcoming this problem, we deposited a series of tapers, either increasing or decreasing in thickness but with the same withdrawal pattern. By comparing the slopes of the increasing and decreasing tapers, some indication of the gravitational effects could be obtained.

With practice and experience, symmetric profiles were eventually obtained. For example, by increasing the rate of change of the withdrawal speed for the lower region of our first lens attempt by $25 \%$ and decreasing the overall withdrawal speed change, as shown in Fig. 7 , the much more acceptable thickness profile illustrated in Fig. 8 was obtained.

\section{Ray Tracing Analysis}

Ray tracing was an extremely useful tool for determining the expected focusing properties of a lens after its thickness profile was determined. Using standard numerical ray tracing methods, ${ }^{17,18}$ we could determine the expected focal length and spot size for various apertures of the lens used by tracing the paths of a

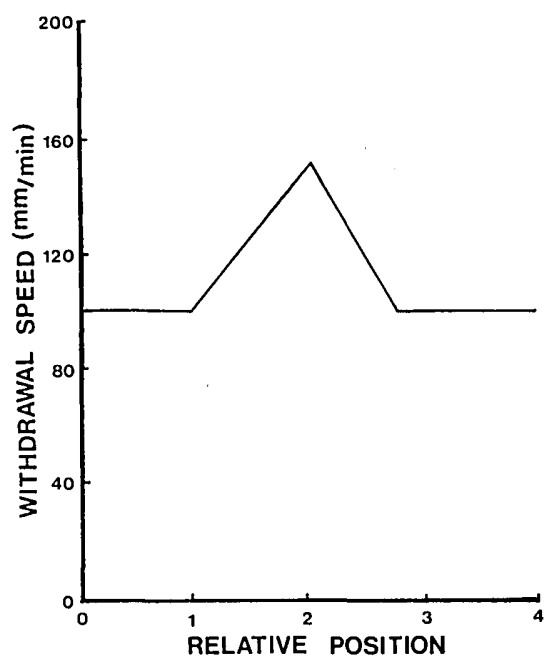

Fig. 7. Modified withdrawal speed pattern.

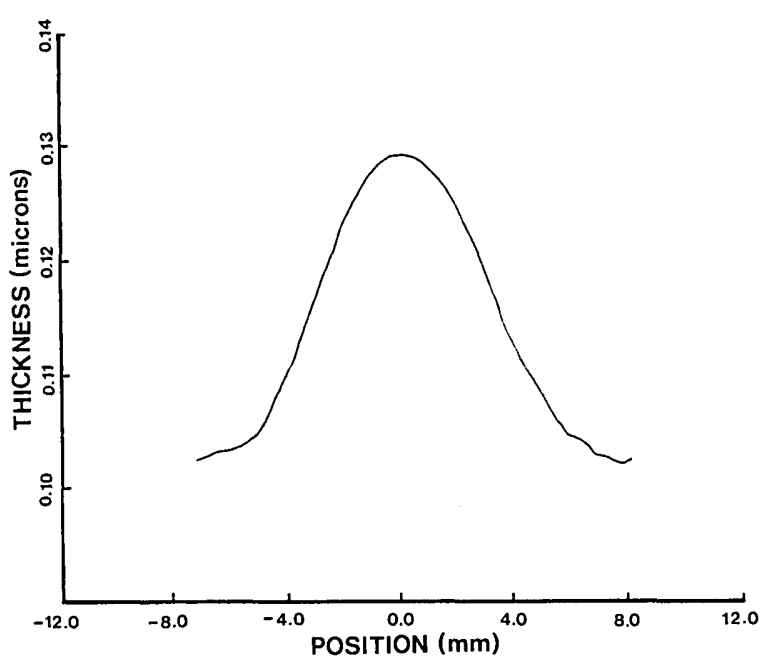

Fig. 8. Improved lens prototype fabricated with the withdrawal speed pattern illustrated in Fig. 7.

collimated group of rays. Here we illustrate this ray tracing analysis for a few of the experimental profiles we have obtained.

Figure 9 illustrates a ray trace through our first lens attempt, whose profile was shown in Fig. 6. The unacceptable focusing resulting from the asymmetric thickness profile is clearly evident. After refining the microprocessor controlled deposition, lenses such as that illustrated in Fig. 8 were obtained. Ray tracing twenty-five rays evenly distributed across a 4-mm aperture of this lens revealed a focal length of $35.69 \mathrm{~mm}$, as shown in Fig. 10. We expect that by reducing the aperture of this lens, the focal length will decrease and the focusing properties will improve even further.

\section{Lens Measurement and Testing}

The measurement and testing of our experimental solution deposited thin film lenses have been carried out with the apparatus illustrated in Fig. 11. Both the 


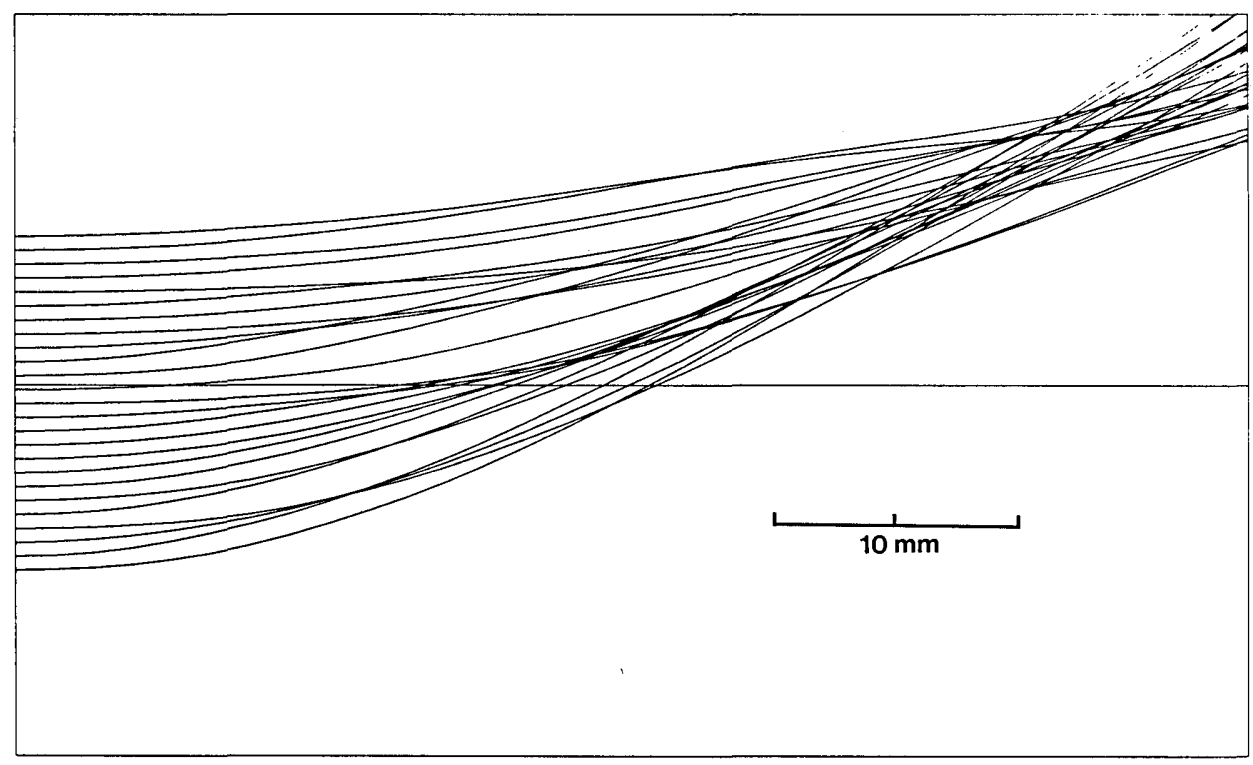

Fig. 9. Ray trace through the first lens attempt illustrated in Fig. 6.

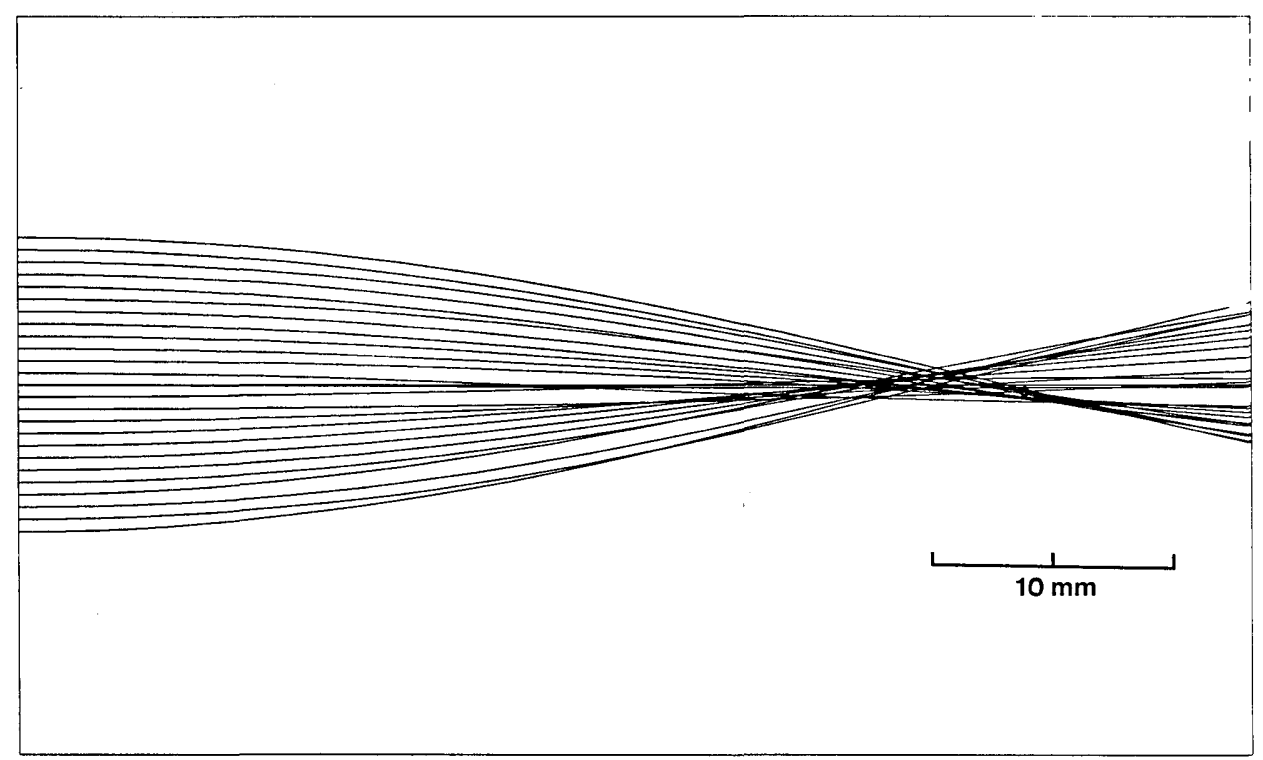

Fig. 10. Ray trace through the lens illustrated in Fig. 8.

measurement of the lens focal length and the scanning of the intensity distribution in the focal plane are possible with this equipment. This apparatus is typical of that used to probe the focal properties of a optical waveguide lens, including the Luneburg lens, geodesic lens, and grating lens. ${ }^{2-5,19}$

\section{A. Lens Scanning Equipment}

Briefly, a polarized $4.0-\mathrm{mW} \mathrm{He}-\mathrm{Ne}$ laser and a $15 \times$ beam expander are used to produce a collimated beam $\sim 20 \mathrm{~mm}$ in diameter. A cylindrical lens with focal length $f=75 \mathrm{~mm}$ reduces this beam to a narrow line. A variable slit is used to select the aperture of the lens. With an aperture of less than $\sim 2 \mathrm{~mm}$, this input beam is approximately uniform. By increasing the slit width or removing the beam expander, a Gaussian input beam is produced. The input beam is coupled to the lens by edge coupling. Yao et al. ${ }^{2}$ have found that, although edge coupling requires a good cleaved edge that is accurately positioned, it is superior to prism coupling in this application, as the use of conventional prism coupling always introduces aberration to the converging or diverging beam.

The guided wave propagates through the lens where it is brought to a focus near the opposite edge. A microscope objective lens serves to reimage and magnify the waveguide edge onto a narrow (3-mm $\times 5-\mu \mathrm{m})$ slit aperture behind which a detector is mounted. The slit width is at least 20 times less than the expected halfwidth of the central lobe of the magnified intensity 


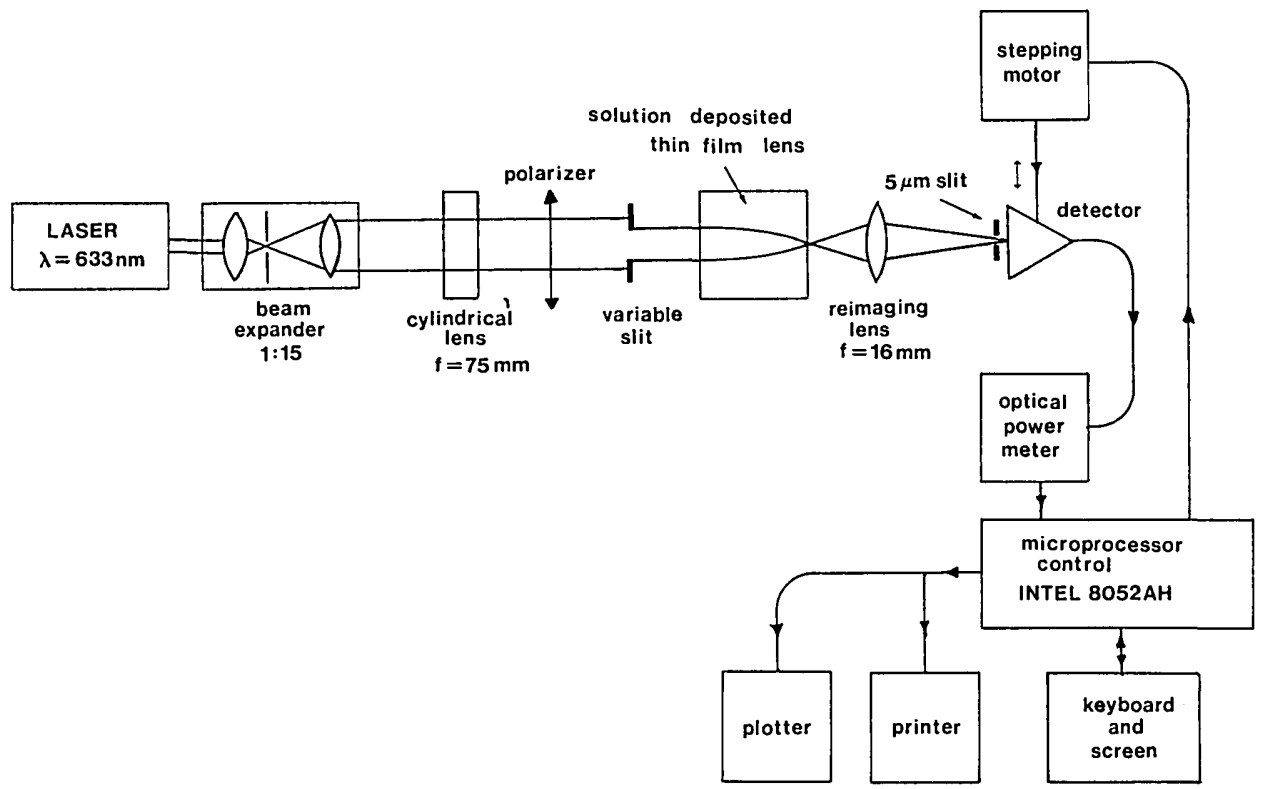

Fig. 11. Schematic diagram of the thin film lens testing arrangement.

pattern in the focal plane. Thus deconvolution of the measured intensity distribution with the slit is not important.

We have used either a $10 \times$ microscope objective with $f / 2$ or a $40 \times$ microscope objective with $f / 0.77$. In this experimental arrangement the measured lens diffraction pattern is the convolution of the true intensity distribution and the reimaging optics unless these microscope objectives exhibit superior performance compared to the lens being tested. The lenses we have designed and fabricated have focal lengths of the order of $20-25 \mathrm{~mm}$ and apertures of up to $5 \mathrm{~mm}$. This corresponds to $f /$ Nos. between $f / 15$ and $f / 4$. When the guided wave is coupled to air from the lens, the $f /$ Nos. are transformed to $f / 9-f / 2.5$, by refraction. Thus only if large experimental lens apertures are used will deconvolution be required.

To determine the optical intensity distribution, the slit and detector are scanned perpendicular to the optic axis of the lens. Scanning is obtained with a stepping motor under microprocessor control, which translates the slit by $2.5 \mu \mathrm{m} / \mathrm{step}$.

\section{B. Experimental Results}

We have chosen a set of three lenses representative of those we have fabricated with $\sim 20-\mathrm{mm}$ focal lengths, for experimental measurement and testing. These lenses are fabricated with different refractive indices and thicknesses and are described in Table I. Two of these lenses, $q 4$ and $r 4$, have a four-layer structure with a profiled layer of higher index deposited on a planar waveguide. The remaining lens, $s 2$, has a three-layer structure with a profiled waveguide serving as both lens and waveguide.

The position of the focal plane is determined experimentally using the method described by Jiang and Ristic. $^{19}$ We consider only the case for which $f>l$, where $f$ is the focal length and $l$ is the length of the lens. This ensures that the focal spot is in the air outside the waveguide. This condition is met by cutting and cleaving the lens so that its length is $1-2 \mathrm{~mm}$ less than the focal length predicted by ray tracing.

Results of the focal length measurements for the three lens prototypes are presented in Table II. These and all other results are for TE guided waves. They show very acceptable agreement between the measured focal length and the focal length determined by ray tracing.

Figure 12 illustrates the experimental lens profile for lens $r 4$ and the theoretical profile which would bring all rays exactly to a point, with focal length $f=$ $21.77 \mathrm{~mm}$. This diagram shows an aperture of $\sim 2.0$ $\mathrm{mm}$ over which there is good agreement between the experimental and theoretical profiles.

Scanning and measurement of the intensity distribution in the focal plane of our experimental lenses

Table I. Lens Materials and Peak Overlay Thickness for the Three Lens Structures Experimentally Measured

\begin{tabular}{ccccc}
\hline & \multicolumn{2}{c}{ Refractive index } & \multicolumn{2}{c}{ Peak thickness $(\mu \mathrm{m})$} \\
Lens & Waveguide & Overlay & Waveguide & Overlay \\
\hline \multirow{2}{*}{$q 4$} & $1.604 \pm 0.005$ & $1.823 \pm 0.005$ & $0.131 \pm 0.006$ & $0.157 \pm 0.001$ \\
$r 4$ & $1.763 \pm 0.003$ & $2.043 \pm 0.094$ & $0.115 \pm 0.006$ & $0.293 \pm 0.003$ \\
$s 2$ & $1.979 \pm 0.090$ & - & $0.146 \pm 0.001$ & - \\
\hline
\end{tabular}

Table II. Comparison of the Theoretical Focal Length, Determined by Ray Tracing and the Experimental Focal Length

\begin{tabular}{ccc}
\hline Lens & $\begin{array}{c}\text { Theoretical } \\
(\mathrm{mm})\end{array}$ & $\begin{array}{c}\text { Experimental } \\
(\mathrm{mm})\end{array}$ \\
\hline$q 4$ & $21.77 \pm 1.89$ & $19.02 \pm 0.05$ \\
$r 4$ & $21.51 \pm 1.32$ & $20.70 \pm 0.05$ \\
$s 2$ & $20.98 \pm 1.84$ & $19.57 \pm 0.05$ \\
\hline
\end{tabular}




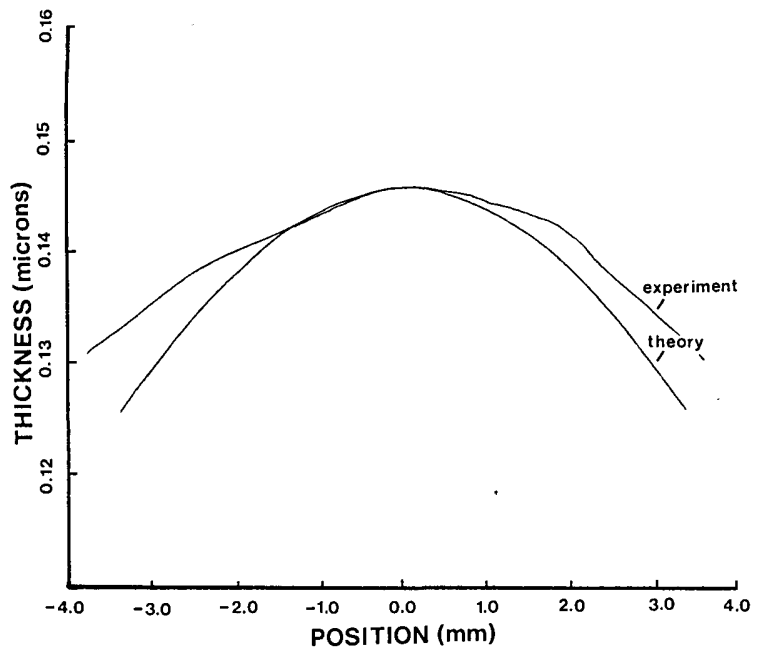

Fig. 12. Experimental and theoretical thickness profiles for lens $r 4$.

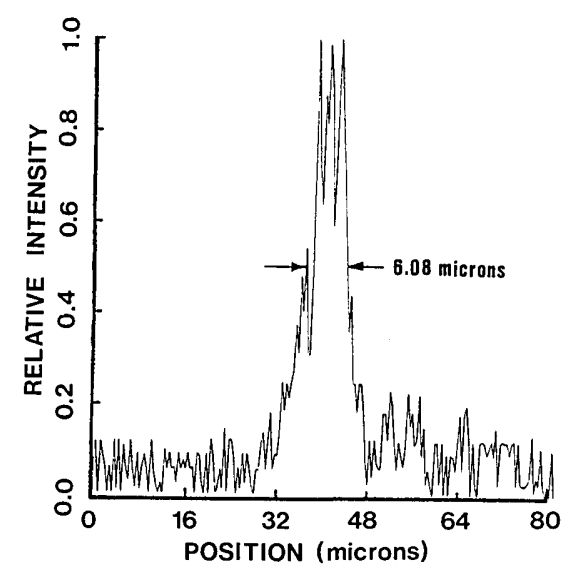

Fig. 13. Intensity distribution in the focal plane of lens $r 4$.

give the best indication of lens performance and is the method of evaluation most often used in the literature. Figure 13 shows the intensity distribution in the focal plane of lens $r 4$. Experimental results for the focal spot width at the $1 / 2$ and $1 / e^{2}$ power levels along with the diffraction limited spot size are presented in Table III. In each case, an input beam $2 \mathrm{~mm}$ in width with a Gaussian distribution was used. The theoretical diffraction limited spot size at the 3-dB power level was determined using

$$
\delta=0.75 \lambda f / a .
$$

Measured spot sizes at the $1 / 2$ power level range from

Table III. Comparison of Measured Focal Spot Size with the Diffraction Limited Spot Size

\begin{tabular}{cccc}
\hline & \multicolumn{2}{c}{ Experimental spot size } & $\begin{array}{c}\text { Diffraction } \\
(\mu \mathrm{m})\end{array}$ \\
Lens & $1 / 2$ & $1 / e^{2}$ & $\begin{array}{c}\text { limited spot size } \\
(\mu \mathrm{m})\end{array}$ \\
\hline$q 4$ & $7.15 \pm 0.07$ & $24.20 \pm 0.07$ & 4.51 \\
$r 4$ & $6.08 \pm 0.16$ & $17.56 \pm 0.06$ & 4.81 \\
$s 2$ & $34.5 \pm 1.00$ & $60.55 \pm 5.00$ & 4.64 \\
\hline
\end{tabular}

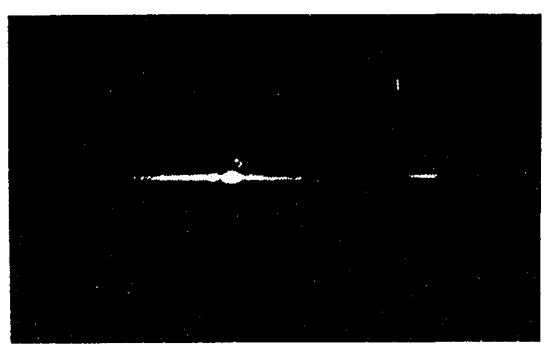

500 microns

Fig. 14. Diffraction pattern of lens $s 2$ magnified by $\sim 58$ times.

1.24 to 7.42 times the diffraction limit. The best lens, $r 4$, had a full width at $1 / 2$ maximum of $6.08 \mu \mathrm{m}$ and a $1 / e^{2}$ width of $17.56 \mu \mathrm{m}$.

The intensity pattern in the focal plane of lens $s 2$ was magnified $\sim 58$ times and projected on a screen to study this pattern more closely. Figure 14 is a photograph of this diffraction pattern, in which the central maximum and secondary maxima are visible. It was interesting to note that only for this lens, $s 2$, which has the largest aberrations, were sidelobes in the lens diffraction pattern clear and well defined. The first two secondary maxima are 6 and $10 \mathrm{~dB}$ below the peak power, and some asymmetry in the diffraction pattern is evident. This asymmetry is most likely a consequence of the asymmetry in the thickness profile of the lens.

For lenses $r 4$ and $q 4$, which outperform lens $s 2$ by a factor of $\sim 5$, we expect that the sidelobes still exist but are lost in the noise surrounding the central maximum. For these lenses, the first secondary maxima should be more than $10 \mathrm{~dB}$ below the peak intensity and are thus beyond the resolution of our scanning apparatus. Furthermore, waveguide losses, in-plane scattering inside the guide, and irregularities in the waveguide edge also contribute to smearing of the sidelobes and the increased noise level.

Finally, it is interesting to compare the performance of the lens we have fabricated with that of other experimental thin film lenses. Our deposition of thickness varying structures from solution is unique. Thus there is no mention in the literature of thin film lenses deposited in this way. However, the Luneburg lens fabricated with a circular profiled overlay layer on a planar waveguide is closely related to the hyperbolic secant lens we have fabricated. Both lenses utilize an effective index gradient to achieve a desired focusing effect. Anderson et $a l .{ }^{4}$ fabricated a thin film Luneburg lens by sputtering through a mask using $\mathrm{Ta}_{2} \mathrm{O}_{5}$ on a 7059 glass waveguide. The lens diameter was 11.2 $\mathrm{mm}$, and the focal length was $12 \mathrm{~mm}$. A Gaussian beam was employed for the input where its width was 2 $\mathrm{mm}$ at the $1 / e^{2}$ point. The measured output beamwidth at the $1 / e^{2}$ point was $11 \mu \mathrm{m}$. The sidelobes were 8-12 dB below the peak intensity but were irregular and showed signs of in-plane scattering. Yao et $a l^{2}{ }^{2}$ fabricated a similar Luneburg lens using a computer simulation model to determine the optimum mask 
shape. In this way a diffraction limited $f / 4$ lens with an 8-mm focal length using a 2-mm aperture was obtained. The measured spot width at the 1/2 power level was $1.6 \mu \mathrm{m}$, and the sidelobes were more than 15 $\mathrm{dB}$ below the focus. Wood et $a .^{3}$ fabricated a novel rectangular Luneburg-type lens by evaporating $\mathrm{As}_{2} \mathrm{~S}_{3}$ through a suitably shaped mask. The lens focal length was $35 \mathrm{~mm}$, and when used at $f / 3.9$ the measured focal spot width was $11.3 \mu \mathrm{m}$ or 1.5 times the diffraction limited value. The central peak was symmetric with the first sidelobes being $6.7 \mathrm{~dB}$ down.

\section{Summary}

This paper has described the development of a solution deposited thin film lens fabricated using a microprocessor controlled dip coating procedure. By carefully and precisely controlling the withdrawal speed of a substrate, from solution we have successfully made optical waveguide lenses which utilize a hyperbolic secant index gradient to provide focusing up to 1.2 times the diffraction limit.

The precise withdrawal speed patterns required to fabricate a hyperbolic secant lens were obtained largely by trial and error, utilizing our prior knowledge of the thin film properties obtained by solution deposition. By successively dip coating lenses, evaluating their profile, and modifying the withdrawal speed pattern, we were able to fabricate symmetric lens profiles. Ray tracing through these structures reveals promising focusing properties. However, the true test of the lens capabilities was in the direct measurement of the focused guided wave.

Three lens prototypes fabricated from different materials were studied in detail. Experimental measurement revealed focal lengths of $19.02-20.70 \mathrm{~mm}$ when the lenses were used with apertures of $2.0 \mathrm{~mm}$. The measured focal lengths agreed well with the predictions of ray tracing. Measurement of the focal spot size revealed spot sizes at the $1 / 2$ power level of 7.15$3.45 \mu \mathrm{m}$, which are equivalent to 1.24-7.42 times the diffraction limit. Analysis of the intensity distribution in the focal plane of these lenses revealed that the sidelobes were $6 \mathrm{~dB}$ below the peak intensity for the lens with the largest aberrations and more than $10 \mathrm{~dB}$ below for the more superior ones.

These lens properties were quite favorable when compared with other thin film lenses. Thus the solution deposited thin film lenses we have fabricated show considerable promise for future applications.

\section{References}

1. F. Zernike, "Luneburg Lens for Optical Waveguide Use," Opt. Commun. 12, 379-381 (1974).
2. S. K. Yao, D. B. Anderson, R. R. August, B. R. Youmans, and C. M. Oania, "Guided-Wave Optical Thin-Film Luneburg Lenses: Fabrication Technique and Properties," Appl. Opt. 18, 40674079 (1979).

3. V. E. Wood, J. R. Busch, D. T. Moore, C. B. Wooley, and W. H. Southwell, "Rectangular Luneburg-Type Lenses for Integrated Optics," Opt. Lett. 8, 226-228 (1983).

4. D. B. Anderson, R. L. Davis, J. T. Boyd, and R. R. August, "Comparison of Optical Waveguide Lens Technologies," IEEE J. Quantum Electron. QE-13, 275-282 (1977).

5. D. A. Bryan, C. R. Chubbs, J. K. Powers, H. E. Tomaschke, W. R. Reed, and E. A. Dalke, "Development of a Tantalum Pentoxide Luneburg Lens," Proc. Soc. Photo-Opt. Instrum. Eng. 321, 2-8 (1982).

6. D. W. Hewak and J. W. Y. Lit, "Fabrication of Tapers and Lenslike Waveguides by a microcontrolled Dip Coating Procedure," Appl. Opt. 28, 4562-4564 (1988).

7. D. W. Hewak and J. W. Y. Lit, "Standardization and Control of a Dip Coating Procedure for Thin Films Deposited from Solution," Can. J. Phys. 66, 861-867 (1988).

8. G. Hatakoshi, H. Inoue, K. Naito, S. Umegaki, and S. Tanaka, "Optical Waveguide Lenses," Opt. Acta 26, 961-968 (1979).

9. D. Mergerian, E. C. Malarkey, R. P. Pautienus, and J. C. Bradely, "Diamond Machined Geodesic Lenses in $\mathrm{LiNbO}_{3}$," Proc. Soc. Photo-Opt. Instrum. Eng. 176, 85-94.

10. A. L. Mikaelian, "Self-Focusing Media with Variable Index of Refraction," Prog. Opt. 17, 283-345 (1980).

11. S. Kawakami and J. Nishizawa, "An Optical Waveguide with the Optimum Distribution of the Refractive Index with Reference to Waveform Distortion," IEEE Trans. Microwave Theory MTT-16, 814-818 (1968).

12. E. G. Rawson, D. R. Herriott, and J. McKenna, "Analysis of Refractive Index Distributions in Cylindrical, Graded-Index Glass Rods (GRIN Rods) Used as Image Relays," Appl. Opt. 9, 753-759 (1970).

13. E. W. Marchand, "Gradient Index Lenses," Prog. Opt. 11, 305337 (1973).

14. K. Tiefenthaler, V. Briguet, E. Buser, M. Horisberger, and W. Lukosz, "Preparation of Planar Optical $\mathrm{SiO}_{2}-\mathrm{TiO}_{2}$ and $\mathrm{LiNbO}_{3}$ Guides with a Dip Coating Method and an Embossing Technique for Fabricating Grating Couplers and Channel Wave Guides," Proc. Soc. Photo-Opt. Instrum. Eng. 401, 165-173 (1983).

15. R. Ulrich and H. P. Weber, "Solution-Deposited Thin Films as Passive and Active Light-Guides," Appl. Opt. 11, 428-434 (1972).

16. K. Heuberger and W. Lubosz, "Embossing Technique for Fabricating Surface Relief Gratings on Hard Oxide Waveguides," Appl. Opt. 25, 1499-1504 (1986).

17. D. W. Hewak and J. W. Y. Lit, "Numerical Ray-Tracing Methods for Gradient Index Media," Can. J. Phys. 63, 234-239 (1985).

18. A. Sharma, D. V. Kumar, and A. K. Ghatak, "Tracing Rays Through Graded-Index Media: A New Method,"Appl. Opt. 21, 984-987 (1982).

19. W. Y. Jiang and V. M. Ristic, "Experimental Study of Anisotropy Dependent Geodesic Lenses," IEEE/OSA J. Lightwave Technol. JT-6, 353-356 (1988).

This work was supported by XISERC of Canada. 